\title{
Appearance and disappearance of thermal renormalons
}

\author{
E. Cavalcanti, ${ }^{1, *}$ J. A. Lourenço, ${ }^{2, \dagger}$ C. A. Linhares, ${ }^{3, \$}$ and A. P. C. Malbouisson ${ }^{1, \S}$ \\ ${ }^{1}$ Centro Brasileiro de Pesquisas Físicas/MCTI, 22290-180 Rio de Janeiro, RJ, Brazil \\ ${ }^{2}$ Departamento de Ciências Naturais, Universidade Federal do Espírito Santo, \\ 29932-540 Campus São Mateus, ES, Brazil \\ ${ }^{3}$ Instituto de Física, Universidade do Estado do Rio de Janeiro, 20559-900 Rio de Janeiro, RJ, Brazil
}

(Received 27 April 2018; published 15 August 2018)

\begin{abstract}
We consider a scalar field model with a $g \phi_{4}^{4}$ interaction and compute the mass correction at next-toleading order in a large- $N$ expansion to study the summability of the perturbative series. It is already known that at zero temperature this model has a singularity in the Borel plane (a "renormalon"). We find that a small increase in temperature adds two countable sets both with an infinite number of renormalons. For one of the sets the position of the poles is thermal independent and the residue is thermal dependent. In the other one both the position of poles and the residues are thermal dependent. However, if we consider the model at extremely high temperatures, such that a dimensional reduction takes place, one observes that all the renormalons disappear and the model becomes Borel summable.
\end{abstract}

DOI: $10.1103 /$ PhysRevD.98.045013

\section{INTRODUCTION}

The understanding of strongly coupled systems remains one of the major challenges in particle physics and requires the knowledge of the nonperturbative regime of quantum chromodynamics (QCD), the currently accepted theory of strong interactions.

Also, in the realm of condensed matter physics, systems involving strongly coupled particles (fermions, for instance) fall, in principle, outside the scope of perturbation theory. However, apart from some simple models, nonperturbative solutions are very hard to be found, which led along the years to attempts to rely in some way on perturbative methods (valid in general for weak couplings) to get some results in strong-coupling regimes [1-6].

It is broadly discussed in the literature whether nonperturbative solutions in field theory can or cannot be recovered from a perturbative expansion. In any case, a procedure is needed to make sense out of the perturbative series. In fact, often the perturbative expansions are asymptotic rather than convergent. Actually, we remember that the perturbative series can be viewed just as a representation of the exact solution and if we want to

\footnotetext{
*erich@cbpf.br

jose.lourenco@ufes.br

*linharescesar@gmail.com

§adolfo@cbpf.br
}

Published by the American Physical Society under the terms of the Creative Commons Attribution 4.0 International license. Further distribution of this work must maintain attribution to the author(s) and the published article's title, journal citation, and DOI. Funded by SCOAP ${ }^{3}$. obtain information about the nonperturbative solution from its perturbative representation some summation technique must be implemented. [1-6]

One of the most employed of these procedures is to investigate, after perturbative renormalization has been performed, the so-called Borel summability of a theory, for a brief introduction see Refs. [7,8] and for a complete review on the subject see Ref. [9]. If we start with an asymptotic series, its Borel transform defines a new series that can be convergent. The representation of the nonperturbative result can be obtained by an inverse Borel transform, essentially a Laplace transform, which requires a contour integration in the complex Borel plane in order to be properly defined. This procedure allows one to gain access to the correct nonperturbative solution in many situations [3]. More precisely: if we take a theory characterized by an already perturbatively renormalized coupling constant $g$ and consider a given quantity $F(g)$ given by a formal series (perhaps asymptotically divergent) in $g, F(g)=$ $\sum_{n} a_{n} g^{n}$; define its Borel transform $\mathcal{B}(F ; b)$ as $\mathcal{B}(F ; b)=$ $\sum_{n} a_{n} b^{n} / n$ ! and the inverse Borel transform as $\tilde{F}(g)=1 / g \int_{0}^{\infty} d b e^{-b / g} \mathcal{B}(F ; b)$. It can be easily verified that $\tilde{F}(g)$ reproduces formally the original series $F(g)$. The interesting point is that even if $F(g)$ is divergent the series $\mathcal{B}(F ; b)$ may converge and in this case the inverse Borel transform $\tilde{F}(g)$ defines a function of $g$ which can be considered in some sense as the sum of the original divergent series $F(g)$. This "mathematical phenomenon" is named Borel summability and is a way of giving a meaning to divergent perturbative series. However, for the inverse Borel transform to be well defined, the absence of singularities at least on the positive real axis of the Borel plane $b$ is required. 
We emphasize that we are working with a model in which the perturbative renormalization of the coupling constant has already been performed, in the spirit of Ref. $[10,11]$. In the above quoted references, by starting with a perturbatively renormalized QFT there can be singularities on the positive real axis of the Borel plane that obstruct the Borel resummation of the perturbation theory. These singularities, in the case of non-asymptotically free theories like $\lambda \phi_{4}^{4}$ and $\mathrm{QED}_{4}$ are called ultraviolet (UV) renormalons.

The study of renormalons is also important from a phenomenological perspective, as one needs, for instance, to know the solution at the nonperturbative level to obtain an estimate of the heavy-quark mass $[12,13]$.

In the context of IR renormalons that arise in the context of asymptotically free theories, it has been a subject of recent investigation to consider compactified theories such as non-Abelian $\mathrm{SU}(\mathrm{N})$ gauge theories on $\mathbb{R}^{3} \times \mathbb{S}^{1}[14]$ and the $\mathbb{C} P^{N-1}$ nonlinear sigma model on $\mathbb{R}^{1} \times \mathbb{S}^{1}[4,15]$. The interest of considering a finite spatial extent $L$ or thermal dependence $\beta=1 / T$ arises from the fact that for small $L$ or large $T$ a weakly coupled regime is observed due to asymptotic freedom $[16,17]$. A careful study of renormalons for an $S U(N)$ gauge theory has been made in Ref. [14]. In it the absence of renormalons is discussed when one introduces a finite small length $L$. However, in the context of non-asymptotically free theories, it is not yet clear how the renormalon phenomenon is influenced by the presence of a finite temperature or finite extension.

In the present article, we investigate the behavior of a scalar field model with $O(N)$ symmetry in four dimensions. Our main concern is the careful investigation of the renormalon poles and residues at next-to-leading order in the large- $N$ expansion, as presented in Sec. II. This $1 / N$ expansion allows to resum a class of diagrams (usually called ring diagrams or necklaces) that generates the renormalon contribution. Following recent literature, we investigate the role of a compactification parameter, here taken as introducing a temperature dependence. First, we review in Sec. III the behavior at zero temperature and find the existence of two renormalons. In Sec. IV, we observe that at small temperatures the system develops a countable set with an infinite number of renormalons that can be separated into two classes: renormalons without thermal poles but that can have thermal residues and renormalons with thermal poles. In Sec. V we consider an extreme increase in temperature, which is related to a dimensional reduction, and obtain that it implies the disappearance of renormalons. We summarize our conclusions in Sec. VI.

\section{SCALAR FIELD MODEL AND RESUMMATION}

We are mainly interested in computing corrections to the field mass in a scalar theory with coupling $(g / N)\left(\phi_{i} \phi_{i}\right)^{2}$, at which $i=1, \ldots, N$. The full propagator $G$ is given by

$$
G=G_{0} \sum_{k=0}^{\infty}\left(\Sigma G_{0}\right)^{k} \equiv \frac{G_{0}}{1-\Sigma G_{0}},
$$

where $\Sigma$ is the sum of all 1PI (one-particle irreducible) diagrams built with the free propagator $G_{0}$. Or, if we establish $\Sigma$ using the full propagator $G$ (a recurrence relation) then, to avoid double counting, it is necessary to consider just the 2PI diagrams. We use a set of 2PI diagrams known as necklace or ring diagrams as illustrated in Fig. 1. This set is the leading order contribution in the $1 / N$ expansion, any other diagram will contribute only at next to leading order in $1 / N$ [18] and is then consistently ignored as a subdominant behavior at this order.

Therefore, at an unspecified spacetime dimension $D$ a necklace with $(k-1)$-pearls is given by $R_{k}(p)$,

$R_{k}(p)=-\frac{g}{N} \int \frac{d^{D} \ell}{(2 \pi)^{D}} \frac{1}{(p-l)^{2}+M^{2}}\left[-\frac{g}{2 N}(N B(\ell))\right]^{k-1}$,

where

$$
B(\ell)=\int \frac{d^{D} q}{(2 \pi)^{D}} \frac{1}{q^{2}+M^{2}} \frac{1}{(q+l)^{2}+M^{2}}
$$

stands for each pearl $[1,18]$.

Thus, by taking into account necklaces with all numbers of pearls we obtain the full correction

$$
\Sigma=\sum_{k=1}^{\infty} R_{k}(p) .
$$

The subsequent analysis of this expression intends to verify whether the series representation is or is not Borel summable. This is entirely dependent on the behavior of $R_{k}(p)$ with respect to the summation index $k$.

Now that we have introduced the general idea, let us investigate the thermal dependence in detail. By making a compactification in imaginary time we introduce the inverse temperature $\beta=1 / T$. With this, the expression for each pearl is modified to

$$
\begin{aligned}
B\left(\ell, \omega_{m}\right)= & \frac{1}{\beta} \sum_{n \in \mathbb{Z}} \int \frac{d^{D-1} q}{(2 \pi)^{D-1}} \frac{1}{q^{2}+\omega_{n}^{2}+M^{2}} \\
& \times \frac{1}{(q+l)^{2}+\left(\omega_{n}-\omega_{m}\right)^{2}+M^{2}},
\end{aligned}
$$

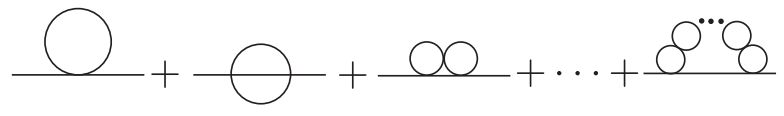

FIG. 1. Sum over the class of necklace diagrams. The case without any pearl is the usual "tadpole" (first diagram), the special case with just one pearl is the usual "sunset" diagram (second diagram). Each vertex contributes a factor $g / N$ and each "pearl" a factor $N$; therefore the whole series has the same order in the $1 / N$ expansion. 
where $\omega_{n}=2 \pi n T$ is the frequency related to the $(D-1)$ dimensional momentum $q$, while the necklaces become

$$
\begin{aligned}
R_{k}\left(p, \omega_{o}\right)= & \frac{(-g)^{k}}{2^{k-1} N \beta} \sum_{m \in \mathbb{Z}} \int \frac{d^{D-1} \ell}{(2 \pi)^{D-1}} \\
& \times \frac{B^{k-1}\left(\ell, \omega_{m}\right)}{(p-l)^{2}+\left(\omega_{o}-\omega_{m}\right)^{2}+M^{2}},
\end{aligned}
$$

where $\omega_{m}$ and $\omega_{o}$ are the frequencies related, respectively, to the loop momentum $\ell$ and the external momentum $p$.

Then, Eq. (5) can be treated by using the Feynman parametrization, integrating over the momenta $q$ and identifying the infinite sum as an Epstein-Hurwitz zeta function $Z^{X^{2}}(\beta ; \nu)$ defined by

$$
Z^{X^{2}}(\beta ; \nu)=\sum_{m \in \mathbb{Z}} \frac{1}{\left(\omega_{m}^{2}+X^{2}\right)^{\nu}}
$$

We now perform the analytic expansion of the EpsteinHurwitz zeta function to whole complex $\nu$ plane [19], which allows us to rewrite Eq. (5) as

$$
\begin{aligned}
B\left(\ell, \omega_{m}\right)= & \frac{\Gamma\left(2-\frac{D}{2}\right)}{(4 \pi)^{\frac{D}{2}}} \int_{0}^{1} d z\left[M^{2}+\left(\ell^{2}+\omega_{m}^{2}\right) z(1-z)\right]^{-2+\frac{D}{2}} \\
& +\frac{1}{(2 \pi)^{\frac{D}{2}}} \sum_{n \in \mathbb{N}^{\star}} \int_{0}^{1} d z \frac{(n \beta)^{2-\frac{D}{2}} \cos [2 \pi n m(1-z)]}{\left[M^{2}+\left(\ell^{2}+\omega_{m}^{2}\right) z(1-z)\right]^{\frac{2-\frac{D}{2}}{2}}} \\
& \times K_{2-\frac{D}{2}}\left[n \beta \sqrt{\left.M^{2}+\left(\ell^{2}+\omega_{m}^{2}\right) z(1-z)\right]}\right.
\end{aligned}
$$

where $K_{\nu}(x)$ is the modified Bessel function of the second kind.

Considering the case where $D=4-2 \varepsilon$, we get

$$
\begin{aligned}
B\left(\ell, \omega_{m}\right)= & B_{0}\left(\ell, \omega_{m}\right)+B_{\beta}\left(\ell, \omega_{m}\right) \\
= & \frac{\Gamma(\varepsilon)}{(4 \pi)^{2}} \int_{0}^{1} d z\left\{1-\varepsilon \ln \left[M^{2}+\left(\ell^{2}+\omega_{m}^{2}\right) z(1-z)\right]\right\} \\
& +\frac{1}{(2 \pi)^{2}} \sum_{n \in \mathbb{N}^{*}} \int_{0}^{1} d z \cos [2 \pi n m(1-z)] \\
& \times K_{0}\left[n \beta \sqrt{M^{2}+\left(\ell^{2}+\omega_{m}^{2}\right) z(1-z)}\right]
\end{aligned}
$$

The temperature-independent component $B_{0}$ is standard and well known [18],

$$
\begin{aligned}
B_{0}\left(\ell, \omega_{m}\right)= & -\frac{1}{\left(4 \pi^{2}\right)}\left\{\ln \frac{M^{2}}{\Lambda^{2}}-2+\sqrt{1+\frac{4 M^{2}}{\ell^{2}+\omega_{m}^{2}}}\right. \\
& \left.\times \ln \left[\frac{1+\sqrt{1+4 \frac{M^{2}}{\ell^{2}+\omega_{m}^{2}}}}{1-\sqrt{1+4 \frac{M^{2}}{\ell^{2}+\omega_{m}^{2}}}}\right]\right\} .
\end{aligned}
$$

For high values of the momentum $\ell$ we have the asymptotic expression

$$
B_{0}\left(\ell, \omega_{m}\right) \sim-\frac{1}{\left(4 \pi^{2}\right)} \ln \frac{\ell^{2}+\omega_{m}^{2}+M^{2}}{M^{2}} .
$$

However, we do not have a solution for the term $B_{\beta}$ for all temperatures. In Secs. IV and V we respectively investigate the regimes of low temperatures and extremely high temperatures.

\section{A FIRST GLANCE: RENORMALON AT $T=0$}

In this section we consider the special case of zero temperature. In this situation the only contribution to the pearl diagram comes from the $B_{0}$ component. To obtain a treatable expression to the necklace diagrams, we consider the expansion for high values of the momentum $\ell$, Eq. (11), at zero temperature

$$
B\left(\ell, \omega_{m}\right) \stackrel{T=0}{\sim}-\frac{1}{(4 \pi)^{2}} \ln \frac{\ell^{2}+M^{2}}{M^{2}} .
$$

At this point we recall that the standard approximation is to consider the leading behavior in the momentum $\ell$, that is, $\ln \left(\ell^{2}+M^{2}\right) \approx \ln \ell^{2}$. Here, we avoid this particular approximation and explore the consequences of keeping the exact term $\ln \left(\ell^{2}+M^{2}\right)$. Let us return to the necklace diagrams. To render the theory finite, we employ the well-established Bogoliubov-Parasiuk-HeppZimmerman (BPHZ) procedure [20] to renormalize the amplitude. Therefore, the renormalized necklace $\hat{R}_{k}(p)$ is

$$
\hat{R}_{k}(p)=R_{k}(p)-R_{k}(0)-\left.p^{2} \frac{\partial}{\partial p^{2}} R_{k}(p)\right|_{p=0} .
$$

We shall drop the hat unless it becomes important to distinguish between the renormalized $\hat{R}_{k}(p)$ and nonrenormalized $R_{k}(p)$ necklaces.

As can be noted, this affects only the $p$-dependent propagator in the zero-temperature version of Eq. (6). Regarding the expression of $R_{k}(p)$ given by Eq. (6), the procedure of Eq. (13) is equivalent to perform the substitution on the denominator 


$$
\begin{aligned}
\frac{1}{p^{2}+\ell^{2}+M^{2}} & \rightarrow \frac{p^{4}}{\left(\ell^{2}+M^{2}\right)^{2}\left(p^{2}+\ell^{2}+M^{2}\right)} \\
& \approx \begin{cases}\frac{p^{4}}{\left(\ell^{2}+M^{2}\right)^{3}}, & \text { low } p ; \\
\frac{p^{2}}{\left(\ell^{2}+M^{2}\right)^{2}}, & \text { high } p,\end{cases}
\end{aligned}
$$

where the standard naive expansion $(p-l)^{2} \approx p^{2}+l^{2}$ is assumed.

In a low- $p$ expansion, then, for $T=0$ and small values of $p$, the integral to be solved to obtain the necklace expression is

$R_{k}(p) \sim-\frac{g p^{4}}{N}\left(\frac{g}{2(4 \pi)^{2}}\right)^{k-1} \int \frac{d^{4} \ell}{(2 \pi)^{4}} \frac{\left(\ln \frac{\ell^{2}+M^{2}}{M^{2}}\right)^{k-1}}{\left(\ell^{2}+M^{2}\right)^{3}}$

To solve it we first perform the integral over the solid angle $\left(\Omega_{4}=2 \pi^{2}\right)$ and then reorganize the result by making the change of variables $\ell^{2}+M^{2}=M^{2} e^{t}$, that is,

$R_{k}(p) \sim-\frac{g p^{4}}{16 \pi^{2} N M^{2}}\left(\frac{g}{2(4 \pi)^{2}}\right)^{k-1} \int_{0}^{\infty} d t\left(e^{-t}-e^{-2 t}\right) t^{k-1}$

At this point we can clearly identify the presence of two gamma functions, so that

$$
\begin{aligned}
R_{k}(p) \sim & -\frac{g p^{4}}{16 \pi^{2} N M^{2}}\left\{(k-1) !\left(\frac{g}{2(4 \pi)^{2}}\right)^{k-1}\right. \\
& \left.-(k-1) !\left(\frac{g}{4(4 \pi)^{2}}\right)^{k-1}\right\}
\end{aligned}
$$

We can finally return to the sum over all contributions Eq. (4),

$$
\Sigma \sim-\frac{2 \tilde{g} p^{2}}{N M^{2}}\left\{\sum_{k=1}^{\infty}(k-1) ! \tilde{g}^{k-1}-\sum_{k=1}^{\infty}(k-1) !\left(\frac{\tilde{g}}{2}\right)^{k-1}\right\},
$$

where we have defined $\tilde{g}=g /\left(2(4 \pi)^{2}\right)$. Both sums are divergent, but we can try to make sense of them by defining a Borel transform,

$$
\begin{aligned}
\mathcal{B}(\Sigma ; y) & \sim-\frac{2 \tilde{g} p^{2}}{N M^{2}}\left\{\sum_{k=1}^{\infty}(\tilde{g} y)^{k-1}-\sum_{k=1}^{\infty}\left(\frac{\tilde{g} y}{2}\right)^{k-1}\right\} \\
& =-\frac{2 \tilde{g} p^{2}}{N M^{2}}\left\{\frac{1}{1-\tilde{g} y}-\frac{1}{1-\tilde{g} y / 2}\right\}
\end{aligned}
$$

We then obtain two poles on the real positive axis of the Borel plane at $y=1 / \tilde{g}, 2 / \tilde{g}$. These poles (renormalons) introduce problems to compute the inverse Borel transform.

In the standard procedure, see Ref. [18], Eq. (15) is solved for very large $\ell$, which is justified as this is the relevant region to get the asymptotic behavior for the $k$ index. This means that the approximation $\left[\ln \left(\ell^{2}+M^{2}\right) / M^{2}\right]^{k-1} /$ $\left(\ell^{2}+M^{2}\right)^{3} \approx\left(\ln \ell^{2}\right)^{k-1} / \ell^{6}$ is employed. Therefore, only the first pole is found (at $y=1 / \tilde{g}$ ) while the second pole is hidden. When $\tilde{g}$ is very small this could be justified as $2 / \tilde{g}$ being very far from the origin.

\section{APPEARANCE OF THERMAL RENORMALONS (LOW TEMPERATURES)}

For low but finite temperatures, we can use the asymptotic representation of the modified Bessel function of the second kind $K_{0}(z) \sim e^{-z} f(z)$, so that the thermal component of the pearl (9) becomes

$$
B_{\beta}\left(\ell, \omega_{m}\right) \sim \frac{1}{(4 \pi)^{2}} \sum_{n \in \mathbb{N}^{\star}} \frac{8 K_{0}(n \beta M)}{n \beta} \frac{1}{\ell^{2}+\omega_{m}^{2}} .
$$

Using the above equation for $B_{\beta}$ and the expression for the $T=0$ component, $B_{0}$ [see Eq. (11)], the quantity $B=B_{0}+B_{\beta}$ can be written in the low-temperature regime as

$$
B\left(\ell, \omega_{m}\right) \sim-\frac{1}{(4 \pi)^{2}}\left[\ln \frac{\ell^{2}+\omega_{m}^{2}+M^{2}}{M^{2}}-\frac{A(\beta)}{\ell^{2}+\omega_{m}^{2}}\right],
$$

where

$$
A(\beta)=\frac{1}{(4 \pi)^{2}} \sum_{n \in \mathbb{N}^{\star}} \frac{8 K_{0}(n \beta M)}{n \beta}
$$

stores information about the dependence on the temperature.

We then replace the expression in Eq. (21) into Eq. (6), employ the BPHZ procedure and use a low- $p$ expansion as in Eq. (14),

$$
\begin{aligned}
R_{k}\left(p, \omega_{o}\right)= & -\frac{g}{N}\left(p^{2}+\omega_{o}^{2}\right)^{2}\left(\frac{g}{2(4 \pi)^{2}}\right)^{k-1} \frac{1}{\beta} \\
& \times \sum_{m \in \mathbb{Z}} \int \frac{d^{3} \ell}{(2 \pi)^{3}} \frac{\left(\ln \frac{\ell^{2}+\omega_{m}^{2}+M^{2}}{M^{2}}-\frac{A(\beta)}{\ell^{2}+\omega_{m}^{2}}\right)^{k-1}}{\left(\ell^{2}+\omega_{m}^{2}+M^{2}\right)^{3}} .
\end{aligned}
$$

So, integrating over the solid angle and expanding the binomial, we get 


$$
\begin{aligned}
R_{k}\left(p, \omega_{o}\right)= & -\frac{g\left(p^{2}+\omega_{o}^{2}\right)^{2}}{2 \pi^{2} N}\left(\frac{g}{2(4 \pi)^{2}}\right)^{k-1} \frac{1}{\beta} \sum_{m \in \mathbb{Z}} \sum_{i=0}^{k-1}\left(\begin{array}{c}
k-1 \\
i
\end{array}\right)(-A(\beta))^{i} \\
& \times \int_{0}^{\infty} d \ell \ell^{2} \frac{\ln ^{k-1-i}\left[\left(\ell^{2}+\omega_{m}^{2}+M^{2}\right) / M^{2}\right]}{\left(\ell^{2}+\omega_{m}^{2}+M^{2}\right)^{3}}\left(\frac{1}{\ell^{2}+\omega_{m}^{2}}\right)^{i} .
\end{aligned}
$$

We reorganize the above expression in a more convenient way to compute the sum over the Matsubara frequencies. The denominator is treated by employing a Feynman parametrization and the logarithm in the numerator is expanded in powers of $\omega_{m}^{2} /\left(\ell^{2}+M^{2}\right)$, which is justified by an asymptotic behavior in $\ell$ assuring that $m / \ell<1$. This allows us to rewrite the above equation in the form

$$
\begin{aligned}
R_{k}\left(p, \omega_{o}\right)= & -\frac{g\left(p^{2}+\omega_{o}^{2}\right)^{2}}{2 \pi^{2} N}\left(\frac{g}{2(4 \pi)^{2}}\right)^{k-1} \sum_{i=0}^{k-1}\left(\begin{array}{c}
k-1 \\
i
\end{array}\right)(-A(\beta))^{i} \int_{0}^{1} d z \frac{\Gamma(3+i) z^{2} z^{i-1}}{\Gamma(3) \Gamma(i)} \\
& \times\left\{\int_{0}^{\infty} d \ell \ell^{2} \ln ^{k-i-1} \frac{\ell^{2}+M^{2}}{M^{2}} \frac{1}{\beta} \sum_{m \in \mathbb{Z}} \frac{1}{\left(\ell^{2}+\omega_{m}^{2}+M^{2} z\right)^{3+i}}\right. \\
& \left.+\int_{0}^{\infty} d \ell \ell^{2} \frac{\ln ^{k-i-2} \frac{\ell^{2}+M^{2}}{M^{2}}}{\ell^{2}+M^{2}} \frac{1}{\beta} \sum_{m \in \mathbb{Z}} \frac{(k-i-1) \omega_{m}^{2}}{\left(\ell^{2}+\omega_{m}^{2}+M^{2} z\right)^{3+i}}+\mathcal{O}\left(\omega_{m}^{4}\right)\right\} .
\end{aligned}
$$

Although we could use this complete expression, this is unnecessary. It can be shown, after a lengthy computation, that the relevant information (poles in the Borel plane) can already be obtained by using the following approximation,

$R_{k}\left(p, \omega_{o}\right) \approx-\frac{g\left(p^{2}+\omega_{o}^{2}\right)^{2}}{2 \pi^{2} N}\left(\frac{g}{2(4 \pi)^{2}}\right)^{k-1} \sum_{i=0}^{k-1}\left(\begin{array}{c}k-1 \\ i\end{array}\right)(-A(\beta))^{i}\left\{\int_{0}^{\infty} d \ell \ell^{2} \ln ^{k-1-i} \frac{\ell^{2}+M^{2}}{M^{2}} \frac{1}{\beta} Z^{\ell^{2}+M^{2}}(\beta ; 3+i)\right\}$,

where $Z^{X^{2}}(\beta ; \nu)$ is the Epstein-Hurwitz zeta function defined in Eq. (7). The contributions of order $\mathcal{O}\left(\omega_{m}^{2}\right)$ do not modify the position of the poles and only change their residues. Moreover, for large values of $k$ the integration of the expression over the Feynman parameter $z$ is asymptotically equal to the expression without the Feynman parameters. To avoid a tedious calculation we do not exhibit in this article the step-by-step of this process.

Taking the approximation in Eq. (26) and considering again the analytic expansion of the Epstein-Hurwitz zeta function to the whole complex $\nu$ plane, we get

$$
\begin{aligned}
R_{k}\left(p, \omega_{o}\right) \approx & -\frac{g\left(p^{2}+\omega_{o}^{2}\right)^{2}}{2 \pi^{2} N}\left(\frac{g}{2(4 \pi)^{2}}\right)^{k-1} \sum_{i=0}^{k-1}\left(\begin{array}{c}
k-1 \\
i
\end{array}\right)(-A(\beta))^{i} \int_{0}^{\infty} d \ell \ell^{2} \ln ^{k-1-i} \frac{\ell^{2}+M^{2}}{M^{2}} \\
& \times \frac{1}{\sqrt{4 \pi} \Gamma(3+i)}\left\{\frac{\Gamma\left(\frac{5}{2}+i\right)}{\left(\ell^{2}+M^{2}\right)^{\frac{5}{2}+i}}+\frac{4}{2^{\frac{5}{2}+i}} \sum_{n \in \mathbb{N}^{*}}\left(\frac{n \beta}{\sqrt{\ell^{2}+M^{2}}}\right)^{\frac{5}{2}+i} K_{\frac{5}{2}+i}\left(n \beta \sqrt{\ell^{2}+M^{2}}\right)\right\} .
\end{aligned}
$$

Since $i$ is an integer, the modified Bessel function of the second kind has a half-integer order, which has the series representation [21]

$$
K_{\frac{5}{2}+i}\left(n \beta \sqrt{\ell^{2}+M^{2}}\right)=\sqrt{\frac{\pi}{2}} \sum_{j=0}^{i+2} \frac{(j+i+2) !}{j !(i+2-j) ! 2^{j}(n \beta)^{j+\frac{1}{2}}} \frac{e^{-n \beta \sqrt{\ell^{2}+M^{2}}}}{\left(\ell^{2}+M^{2}\right)^{j+\frac{1}{2}}} .
$$

So, the remaining integrals are given by 


$$
\begin{aligned}
R_{k}\left(p, \omega_{o}\right)= & -\frac{g\left(p^{2}+\omega_{o}^{2}\right)^{2}}{4 \pi^{5 / 2} N}\left(\frac{g}{2(4 \pi)^{2}}\right)^{k-1} \sum_{i=0}^{k-1}\left(\begin{array}{c}
k-1 \\
i
\end{array}\right) \\
& \times \frac{(-A(\beta))^{i}}{(2+i) !}\left\{\Gamma\left(\frac{5}{2}+i\right) \int_{0}^{\infty} d \ell \ell^{2} \frac{\ln ^{k-1-i} \frac{\ell^{2}+M^{2}}{M^{2}}}{\left(\ell^{2}+M^{2}\right)^{\frac{5}{2}+i}}\right. \\
& +\frac{\sqrt{\pi}}{2^{1+i}} \sum_{n \in \mathbb{N}^{*}} \sum_{j=0}^{i+2} \frac{(j+i+2) !}{j !(i+2-j) ! 2^{j}}(n \beta)^{j-i-2} \\
& \left.\times \int_{0}^{\infty} d \ell \ell^{2} \frac{e^{-n \beta \sqrt{\ell^{2}+M^{2}}}}{\left(\sqrt{\ell^{2}+M^{2}}\right)^{2 j+i+\frac{7}{2}}} \ln ^{k-1-i} \frac{\ell^{2}+M^{2}}{M^{2}}\right\} .
\end{aligned}
$$

The first integral in the preceding equation can be solved as in the zero-temperature case (see Sec. III) by the change of variables $\ell^{2}+M^{2}=M^{2} e^{t}$. One must note that $\sqrt{e^{t}-1}$ has an upper bound $\sqrt{e^{t}}$ that is also its asymptotic value for large values of the momentum $t$ (which means also large values of the index $k$ ). Then, we can use that $\sqrt{e^{t}-1} \lesssim \sqrt{e^{t}}$ to simplify the integral

$$
\begin{aligned}
\mathcal{I}_{1} & =\int_{0}^{\infty} d \ell \ell^{2} \frac{\ln ^{k-1-i} \frac{\ell^{2}+M^{2}}{M^{2}}}{\left(\ell^{2}+M^{2}\right)^{\frac{5}{2}+i}} \\
& =\frac{1}{2 M^{2+2 i}} \int_{0}^{\infty} d t t^{k-i-1} \sqrt{e^{t}-1} e^{-t\left(\frac{3}{2}+i\right)} \\
& \lesssim \frac{1}{2 M^{2+2 i}} \int_{0}^{\infty} d t t^{k-i-1} e^{-t(1+i)} \\
& =\frac{(k-i-1) !}{2 M^{2+2 i}} \frac{1}{(1+i)^{k-i}} .
\end{aligned}
$$

For the second integral in Eq. (29) we make the change of variables $\ell^{2}+M^{2}=M^{2} r^{2}$ so that we obtain

$$
\begin{aligned}
\mathcal{I}_{2} & =\int_{0}^{\infty} d \ell \ell^{2} \frac{e^{-n \beta \sqrt{\ell^{2}+M^{2}}}}{\left(\sqrt{\ell^{2}+M^{2}}\right)^{2 j+i+\frac{7}{2}}} \ln ^{k-1-i} \frac{\ell^{2}+M^{2}}{M^{2}} \\
& =2^{k-i-1} M^{3-2(j+i / 2+7 / 4)} \int_{1}^{\infty} d r \sqrt{r^{2}-1} \frac{e^{-n \beta r} \ln ^{k-i-1} r}{r^{2(j+i / 2+7 / 4)-1}} \\
& \lesssim 2^{k-i-1} M^{3-2(j+i / 2+7 / 4)} \int_{1}^{\infty} d r \frac{e^{-n \beta r} \ln ^{k-i-1} r}{r^{2(j+i / 2+7 / 4)-2}} .
\end{aligned}
$$

Once more, we used that $\sqrt{r^{2}-1} \lesssim r$, which considerably simplifies the integral and allows it to be identified as the Milgram generalization of the integroexponential function whose asymptotic behavior is known [22]

$$
\begin{aligned}
E_{s}^{\alpha}(z) & =\frac{1}{\Gamma(\alpha+1)} \int_{1}^{\infty} d t \frac{(\ln t)^{\alpha} e^{-z t}}{t^{s}} \\
& \stackrel{\operatorname{Re} z \rightarrow \infty}{\sim} \frac{e^{-z}}{z^{\alpha+1}}\left[1-\frac{(\alpha+1)(\alpha+2 s)}{2 z}+\cdots\right] .
\end{aligned}
$$

Hence, after substituting Eqs. (30) and (31) into Eq. (29), and using the asymptotic behavior of the generalized integroexponential, Eq. (32), we have

$$
\begin{aligned}
R_{k}\left(p, \omega_{o}\right) \lesssim & -\frac{g\left(p^{2}+\omega_{o}^{2}\right)^{2}}{4 \pi^{5 / 2} N}\left(\frac{g}{2(4 \pi)^{2}}\right)^{k-1} \sum_{i=0}^{k-1}\left(\begin{array}{c}
k-1 \\
i
\end{array}\right) \\
& \times \frac{(-A(\beta))^{i}(k-i-1) !}{(2+i) !}\left\{\Gamma\left(\frac{5}{2}+i\right) \frac{1}{2 M^{2+2 i}}\right. \\
& \times \frac{1}{(1+i)^{k-i}}+\sqrt{\pi} \sum_{n \in \mathbb{N}^{*}} \sum_{j=0}^{i+2} \frac{(j+i+2) !}{j !(i+2-j) !} \\
& \left.\times \frac{2^{k-2 i-j-2}}{(n \beta)^{k+j-2 i-2}} \frac{e^{-n \beta}}{M^{2 j+i+1 / 2}}\right\}
\end{aligned}
$$

Now, let us focus attention on the $k$-dependence. The previous equation can then be rewritten as

$R_{k} \lesssim \gamma_{1} \tilde{g}^{k-1}(k-1) ! \sum_{i=0}^{k-1}\left[\frac{\gamma_{2, i}(\beta, M)}{(1+i)^{k-1}}+\sum_{n \in \mathbb{N}^{\star}} \frac{2^{k-1} \gamma_{3, i, n}(\beta, M)}{(n \beta)^{k-1}}\right]$

where we have defined,

$$
\begin{aligned}
& \gamma_{1}=-\frac{g\left(p^{2}+\omega_{o}^{2}\right)^{2}}{4 \pi^{5 / 2} N}, \\
& \tilde{g}=\frac{g}{2(4 \pi)^{2}},
\end{aligned}
$$

$$
\begin{aligned}
\gamma_{2, i}(\beta, M)= & \frac{(-A(\beta))^{i}}{(2+i) ! i !} \Gamma\left(\frac{5}{2}+i\right) \frac{(1+i)^{i-1}}{2 M^{2+2 i}}, \\
\gamma_{3, i, n}(\beta, M)= & \sum_{j=0}^{i+2} \frac{(-A(\beta))^{i}}{(2+i) ! i !} \sqrt{\pi} \frac{(j+i+2) !}{j !(i+2-j) !} \\
& \times \frac{e^{-n \beta}}{M^{2 j+i+1 / 2}} \frac{2^{-2 i-j-1}}{(n \beta)^{j-2 i-1}} .
\end{aligned}
$$

The sum over all necklaces is then

$$
\begin{aligned}
\mathcal{R}= & \sum_{k \in \mathbb{N}^{\star}} R_{k} \lesssim \sum_{k \in \mathbb{N}^{\star}} \gamma_{1} \tilde{g}^{k-1}(k-1) ! \sum_{i=0}^{k-1}\left[\frac{\gamma_{2, i}(\beta, M)}{(1+i)^{k-1}}\right. \\
& \left.+\sum_{n \in \mathbb{N}^{\star}} \frac{2^{k-1}}{(n \beta)^{k-1}} \gamma_{3, i, n}(\beta, M)\right] .
\end{aligned}
$$


The range of summation for the double sum is $0 \leq i<k<\infty$; we can change the sum ordering and then split the sum over the index $k$ in the form

$$
\sum_{k \in \mathbb{N}^{+}} \sum_{i=0}^{k-1} f_{i, k}=\sum_{i=0}^{\infty} \sum_{k=i+1}^{\infty} f_{i, k}=\sum_{i=0}^{\infty}\left(\sum_{k=1}^{\infty} f_{i, k}-\sum_{k=1}^{i+1} f_{i, k}\right) .
$$

The first double sum has the dominant contribution; this can be seen by checking for each value of $i$. Therefore, the relevant contribution is

$$
\begin{aligned}
\mathcal{R} \sim \gamma_{1} & \sum_{i=0}^{\infty}\left[\gamma_{2, i} \sum_{k=1}^{\infty} \frac{\tilde{g}^{k-1}(k-1) !}{(1+i)^{k-1}}\right. \\
& \left.+\sum_{n \in \mathbb{N}^{*}} \gamma_{3, i, n} \sum_{k=1}^{\infty} \frac{\tilde{g}^{k-1}(k-1) !}{(n \beta / 2)^{k-1}}\right] .
\end{aligned}
$$

However, this is not summable due to the presence of the $(k-1)$ !. To overcome this difficulty we can employ a Borel transform,

$$
\begin{aligned}
\mathcal{B}(\mathcal{R} ; y) \sim \gamma_{1} \sum_{i=0}^{\infty}\left[\gamma_{2, i} \sum_{k=1}^{\infty} \frac{\tilde{g}^{k-1} y^{k-1}}{(1+i)^{k-1}}\right. \\
\left.+\sum_{n \in \mathbb{N}^{*}} \gamma_{3, i, n} \sum_{k=1}^{\infty} \frac{\tilde{g}^{k-1} y^{k-1}}{(n \beta / 2)^{k-1}}\right] \\
=\gamma_{1} \sum_{i=0}^{\infty}\left[\frac{\gamma_{2, i}(\beta, M)}{1-\frac{\tilde{g} y}{1+i}}+\sum_{n \in \mathbb{N}^{*}} \frac{\gamma_{3, i, n}(\beta, M)}{1-\frac{2 \tilde{g} y}{n \beta}}\right] .
\end{aligned}
$$

Finally, we see in Eq. (37) the renormalons that appear at low temperatures. There are two different sets of renormalons both with residues that are thermal dependent, respectively $\gamma_{2, i}(\beta, M)$ and $\gamma_{3, i, n}(\beta, M)$. The first set of renormalons was already found in previous works [23]; it is characterized by poles whose position are thermalindependent and they are located along the real axis at positions $(1+i) / \tilde{g}$ for $i \in \mathbb{N}$. However, the second set of poles, as far as we know, has not yet been reported. These poles are also in the real axis but they are thermaldependent as they are located at $n \beta / 2 \tilde{g}$ for $n \in \mathbb{N}^{\star}$. The existence of this new set seems to be a remarkable enrichment for the model.

We remark that in the limit of extremely small temperatures these new renormalons are all very far from the origin and this may justify why they are usually hidden. Therefore, our result can be viewed as a first correction to the standard approach. Furthermore, as we pointed out before, we claim that our approximation in Eq. (26) is the sufficient one (at least to describe the poles) and any further corrections shall only change the residues. This means that we have mapped all the renormalons that appear at low temperatures.
As a further comment, we remember that in Sec. III we show that at zero temperature there is a hidden second pole located at $2 / \tilde{g}$. This does not add any new poles at low temperatures because, as can be easily noted, we already have an infinite set of poles located at $i / \tilde{g}$ for $i \in \mathbb{N}^{\star}$.

\section{DISAPPEARANCE OF THERMAL RENORMALONS (EXTREMELY HIGH TEMPERATURES)}

In this section we explore the regime of extremely high temperatures. In fact, we consider the regime of temperatures $T \rightarrow \infty$ which is equivalent to a dimensional reduction of one unit. In this case, it is well-known that if the original theory was UV divergent the resulting theory is not anymore UV divergent; which means that UV renormalons should disappear. In this work, we recover this fact using our formalism. In this situation, to treat Eq. (9) we can use the following series expansion of the modified Bessel function of the second kind [21],

$$
K_{0}(z)=-\ln \frac{z e^{\gamma}}{2}-\frac{z^{2}}{4} \ln \frac{z e^{\gamma-1}}{2}+\mathcal{O}\left(z^{4}\right)
$$

The result is easier to get by assuming from the beginning that $m=0$ (which means that this is the only relevant mode) and recalling the following properties of the Riemann zeta function, $\zeta(s)=\sum_{n \in \mathbb{N}^{\star}} n^{-s}$,

$$
\begin{aligned}
\zeta^{\prime}(s) & =-\sum_{n \in \mathbb{N}^{+}} \frac{\ln n}{n^{s}}, \\
\zeta(0) & =-1 / 2, \\
\zeta^{\prime}(0) & =\ln \sqrt{2 \pi}, \\
\zeta(-2 k) & =0, \quad \forall k \in \mathbb{N}^{\star}, \\
\zeta^{\prime}(-2 k) & =\frac{(-1)^{k} \zeta(2 k+1)(2 k) !}{2^{2 k+1} \pi^{2 k}}, \quad \forall k \in \mathbb{N}^{\star} .
\end{aligned}
$$

Remembering Eq. (9), we then obtain the result

$$
\begin{aligned}
B_{\beta}\left(\ell, \omega_{m}\right) \sim & -B_{0}\left(\ell, \omega_{m}\right)-\frac{1}{8 \pi^{2}} \ln \frac{4 \pi T}{M e^{\gamma}} \\
& -\frac{\zeta(3)}{2^{7} \pi^{4}}\left(M^{2}+\frac{\ell^{2}}{6}\right) \frac{1}{T^{2}},
\end{aligned}
$$

revealing that at extremely high temperatures the original contribution from zero temperature is not present anymore. This has a major impact and is responsible for the disappearance of the renormalons. Therefore, we may write 


$$
B\left(\ell, \omega_{m}\right)^{T \rightarrow \infty}-\frac{1}{8 \pi^{2}} \ln \frac{4 \pi T}{M e^{\gamma}}-\frac{\zeta(3)}{2^{7} \pi^{4}}\left(M^{2}+\frac{\ell^{2}}{6}\right) \frac{1}{T^{2}} .
$$

If we replace this back into the necklace expression $R_{k}(p, o)$, in Eq. (23), we get

$$
\begin{aligned}
R_{k}\left(p, \omega_{o}\right)= & -g\left(p^{2}+\omega_{o}\right)^{2}\left(\frac{g}{2(4 \pi)^{2} N}\right)^{k-1} \frac{1}{\beta} \\
& \times \sum_{n \in \mathbb{Z}} \int \frac{d^{3} \ell}{(2 \pi)^{3}} \frac{\ln ^{k-1}\left(\frac{4 \pi T}{M e^{\gamma}}\right)}{\left(\ell^{2}+\omega_{n}^{2}+M^{2}\right)^{3}} .
\end{aligned}
$$

Since the integration over the internal loop is independent of $k$ we find that

$$
R_{k} \propto\left(2 \tilde{g} \ln \left(\frac{4 \pi T}{M e^{\gamma}}\right)\right)^{k-1}
$$

and, therefore, there is no renormalon in this case.

The function $\Sigma(g)=\sum_{k=1}^{\infty} R_{k}(p)$ is Borel summable,

$$
\Sigma \sim \frac{1}{1-2 \tilde{g} \ln \left(\frac{4 \pi T}{M e^{\gamma}}\right)}
$$

it is a meromorphic function of the coupling constant $\tilde{g}$ having a simple pole at $\tilde{g}=\left[2 \ln \left(4 \pi T /\left(M e^{\gamma}\right)\right)\right]^{-1}$.

\section{CONCLUSION}

In this article we study the existence of renormalons in a scalar field theory with a $g \phi_{4}^{4}$ coupling at next-to-leading order in a large- $N$ expansion. The results in the literature report that there is one renormalon pole at zero temperature (located at $y=1 / \tilde{g}$ ) and there is an appearance of a countable infinite set of renormalons at low temperatures with the property that the poles are thermal-independent (located at $y=i / \tilde{g}$, for $i \in \mathbb{N}^{\star}$ ). Although, in this article, the standard behavior is reproduced, we also manage to identify the existence of hidden poles, both at zero temperature and at low temperatures. As far as we know, it seems that this fact has not been noted in the literature. Perhaps, these poles were hidden by the approximations used. The extra pole at zero temperature is slightly shifted on the real axis $(y=2 / \tilde{g})$ and can be ignored, as it is done currently in the literature, if the coupling is small enough. At low temperatures, however, there is an entirely new set of renormalons on the real axis that are located at $y=n \beta / 2 \tilde{g}$ for $n \in \mathbb{N}^{\star}$. The appearance of renormalons with a small increase in temperature is a remarkable feature of the theory. In this paper we claim that we have mapped all the poles that occur at low temperatures, therefore identifying completely the thermal renormalons that appear. Any further approximation would only improve the value of the residues, but would not modify the number nor the position of the poles in the Borel plane.

Furthermore, we obtain that at extremely high temperatures, which is related to a dimensional reduction from $D=$ 4 to $D=3$, as expected, no renormalon singularities occur and the series becomes Borel summable. This seems to indicate that we could speculate about the existence of a "critical temperature" at which renormalons appear/ disappear. This will be the subject of investigation in future work.

\section{ACKNOWLEDGMENTS}

The authors thank the Brazilian agency Conselho Nacional de Desenvolvimento Científico e Tecnológico (CNPq) for partial financial support.
[1] Large Order Behavior of Perturbation Theory, edited by J.C. Le Guillou and J. Zinn-Justin (North Holland, Amsterdam, 2013), p. 580.

[2] I. Aniceto and R. Schiappa, Commun. Math. Phys. 335, 183 (2015).

[3] A. Grassi, M. Marino, and S. Zakany, J. High Energy Phys. 05 (2015) 038.

[4] A. Cherman, D. Dorigoni, and M. Ünsal, J. High Energy Phys. 10 (2015) 056.

[5] D. Dorigoni, arXiv:1411.3585.

[6] C. M. Bender and S. A. Orszag, Advanced Mathematical Methods for Scientists and Engineers I (Springer, New York, 1999).

[7] V. Rivasseau, From Perturbative to Constructive Renormalization (Princeton University Press, Princeton, NJ, 1991).

[8] F. C. Khanna, A. P. C. Malbouisson, J. M. C. Malbouisson, and A. R. Santana, Thermal Quantum Field Theory-Algebraic
Aspects and Applications (World Scientific Publishing, Singapore, 2009).

[9] B. Hirsbrunner, La sommabilité selon Borel et son application en théorie des champs, Ph.D. thesis, Université de Lausanne-Faculté des Sciences, 1981.

[10] F. David, J. Feldman, and V. Rivasseau, Commun. Math. Phys. 116, 215 (1988).

[11] G. 't Hooft, The Whys of Subnuclear Physics, The Subnuclear Series Vol. 15, edited by A. L. Zichichi (Springer, New York, 1979), p. 943.

[12] M. Shifman, Int. J. Mod. Phys. A 30, 1543001 (2015).

[13] M. Beneke and V. M. Braun, Nucl. Phys. B426, 301 (1994).

[14] M. M. Anber and T. Sulejmanpasic, J. High Energy Phys. 01 (2015) 139.

[15] M. Shifman and M. Ünsal, Phys. Rev. D 78, 065004 (2008).

[16] A. P. C. Malbouisson, J. M. C. Malbouisson, A. E. Santana, and J. C. da Silva, Phys. Lett. B 583, 373 (2004). 
[17] F. C. Khanna, A. P. C. Malbouisson, J. M. C. Malbouisson, and A. E. Santana, Phys. Rev. D 85, 085015 (2012).

[18] J. Zinn-Justin, Quantum Field Theory and Critical Phenomena, 4th ed. (Clarendon Press, Oxford, 2002).

[19] E. Elizalde, Ten Physical Applications of Spectral Zeta Functions, 2nd ed. (Springer-Verlag, Berlin Heidelberg, 1995).
[20] C. Itzykson and J. B. Zuber, Physics (McGraw-Hill, New York, 1980).

[21] I. S. Gradshteyn and I. M. Ryzhik, Table of Integrals, Series, and Products, 7th ed. (Academic Press, New York, 2007).

[22] M. S. Milgram, Math. Comput. 44, 443 (1985).

[23] M. Loewe and C. Valenzuela, Mod. Phys. Lett. A 15, 1181 (2000). 